\title{
Anaesthesia for caesarean section and postpartum cardiovascular events in congenital heart disease: a retrospective cohort study
}

\author{
Akito Tsukinaga $^{1}$, Kenji Yoshitani ${ }^{1}$, Yosuke Kubota ${ }^{1}$, Eiki Kanemaru ${ }^{1}$, Kunihiro \\ Nishimura $^{1}$, Michikazu Nakai ${ }^{1}$, Soshiro Ogata ${ }^{1}$, Rie Tsukinaga ${ }^{1}$, Chizuko Kamiya ${ }^{1}$, Jun \\ Yoshimatsu $^{1}$, and Yoshihiko Ohnishi ${ }^{1}$ \\ ${ }^{1}$ National Cerebral and Cardiovascular Center
}

September 21, 2020

\begin{abstract}
Objective To clarify the association between anaesthetic technique and maternal and neonatal outcomes in parturients with CHD. Design Retrospective observational cohort study. Setting Academic hospital. Population A total of 263 consecutive parturients with congenital heart disease (CHD) who underwent caesarean section. Methods Charts from 1994-2019 were reviewed. Main outcome measures We compared postpartum cardiovascular events (composite of heart failure, pulmonary hypertension, arrhythmia, and thromboembolic complications) and neonatal outcomes (intubation and Apgar score $<7$ at 1 or 5 minutes) by anaesthetic technique. Results Among 263 caesarean sections, general anaesthesia was performed in 47 $(17.9 \%)$ parturients and neuraxial anaesthesia in $214(81.3 \%)$ parturients. Cardiovascular events were more common in the general anaesthesia group $(n=7 ; 14.9 \%)$ than in the neuraxial anaesthesia group $(n=17 ; 7.9 \%)$. Generalized linear mixed models assuming a binomial distribution (i.e., mixed-effects logistic regression) with a random intercept for each modified World Health Organization classification for maternal cardiovascular risk revealed that general anaesthesia is not significantly associated with cardiovascular events (odds ratio [OR], 1.00; 95\% confidence interval [CI], 0.30-3.29). In addition, general anaesthesia was associated with composite neonatal outcomes (Apgar score $<7$ at 1 or 5 minutes or need for neonatal intubation; OR, 13.3; $95 \%$ CI, 5.52-32.0). Conclusion Anaesthetic technique is not significantly associated with postpartum composite cardiovascular events. General anaesthesia is significantly associated with increased need for neonatal intubation and lower Apgar scores. Funding No external funding was used in the conduct of this study. Keywords Anaesthesia, caesarean section, postpartum, cardiovascular events, congenital heart disease, neonatal outcomes
\end{abstract}

Anaesthesia for caesarean section and postpartum cardiovascular events in congenital heart disease: a retrospective cohort study

A Tsukinaga, ${ }^{\mathrm{a}}$ K Yoshitani, ${ }^{\mathrm{a}}$ Y Kubota, ${ }^{\mathrm{a}}$ E Kanemaru, ${ }^{\mathrm{a}} \mathrm{K}$ Nishimura, ${ }^{\mathrm{b}} \mathrm{M}$ Nakai, ${ }^{\mathrm{c}}$ S Ogata, ${ }^{\mathrm{b}} \mathrm{R}$ Tsukinaga, ${ }^{\mathrm{d}}$ CA Kamiya, ${ }^{\mathrm{d}}$ J Yoshimatsu, ${ }^{\mathrm{d}} \mathrm{Y} \mathrm{Ohnishi}^{\mathrm{a}}$

a Department of Anaesthesiology, National Cerebral and Cardiovascular Centre, Suita, Osaka, Japan

${ }^{\mathrm{b}}$ Department of Preventive Medicine and Epidemiology, National Cerebral and Cardiovascular Centre, Suita, Osaka, Japan

${ }^{\mathrm{c}}$ Department of Statistical Analysis, National Cerebral and Cardiovascular Centre, Suita, Osaka, Japan

d Department of Obstetrics and Gynaecology, National Cerebral and Cardiovascular Centre, Suita, Osaka, Japan

Corresponding Author:

Kenji YOSHITANI, MD 
Department of Anaesthesiology, Department of Transfusion, National Cerebral and Cardiovascular Centre, 6-1 Kishibe-shinmachi, Suita, Osaka, Japan

TEL: +81-6-6170-1070

E-mail:ykenji@ncvc.go.jp

Running head: Anaesthesia for caesarean section in CHD

Abstract

Objective To clarify the association between anaesthetic technique and maternal and neonatal outcomes in parturients with CHD.

Design Retrospective observational cohort study.

Setting Academic hospital.

Population A total of 263 consecutive parturients with congenital heart disease (CHD) who underwent caesarean section.

Methods Charts from 1994-2019 were reviewed.

Main outcome measures We compared postpartum cardiovascular events (composite of heart failure, pulmonary hypertension, arrhythmia, and thromboembolic complications) and neonatal outcomes (intubation and Apgar score $<7$ at 1 or 5 minutes) by anaesthetic technique.

Results Among 263 caesarean sections, general anaesthesia was performed in 47 (17.9\%) parturients and neuraxial anaesthesia in $214(81.3 \%)$ parturients. Cardiovascular events were more common in the general anaesthesia group $(n=7 ; 14.9 \%)$ than in the neuraxial anaesthesia group $(n=17 ; 7.9 \%)$. Generalized linear mixed models assuming a binomial distribution (i.e., mixed-effects logistic regression) with a random intercept for each modified World Health Organization classification for maternal cardiovascular risk revealed that general anaesthesia is not significantly associated with cardiovascular events (odds ratio [OR], 1.00; 95\% confidence interval [CI], 0.30-3.29). In addition, general anaesthesia was associated with composite neonatal outcomes (Apgar score $<7$ at 1 or 5 minutes or need for neonatal intubation; OR, 13.3; 95\% CI, 5.52-32.0).

Conclusion Anaesthetic technique is not significantly associated with postpartum composite cardiovascular events. General anaesthesia is significantly associated with increased need for neonatal intubation and lower Apgar scores.

Funding No external funding was used in the conduct of this study.

Keywords Anaesthesia, caesarean section, postpartum, cardiovascular events, congenital heart disease, neonatal outcomes

Tweetable abstract Anaesthetic technique for caesarean section is not significantly associated with cardiovascular events in patients with CHD.

\section{Introduction}

Given the advances in the management of congenital heart disease (CHD) during the last few decades, it has become common for women with CHD to become pregnant. ${ }^{1,2}$ Pregnancy causes tremendous changes in hemodynamics ${ }^{3}$ and could result in decreased cardiac function particularly in women with CHD. Compared with the general population, a higher proportion of women with CHD undergo caesarean section for cardiac indications. ${ }^{4,5}$

In pregnant women without heart disease, neuraxial anaesthesia is the preferred technique for caesarean section due to difficulties in airway management and the desire to minimize the use of systemic medications that can be transferred to the fetus. ${ }^{6-8}$ For pregnant women with heart disease or CHD, neuraxial anaesthesia is usually favoured over general anesthesia. ${ }^{9-12}$ However, there have been no randomized controlled 
studies about which anaesthetic technique is superior in parturients with high cardiac risk. A systematic review of case reports suggest higher maternal mortality with general anaesthesia and improved outcomes with neuraxial anaesthesia, but this systematic review could not exclude selection bias due to sicker parturients receiving general anesthesia. ${ }^{13}$ Some parturients with CHD cannot avoid general anaesthesia because of contraindications to neuraxial anaesthesia, emergent surgery, or need for invasive monitoring. Therefore, clarifying the effect of these anaesthetic techniques might help reduce maternal and neonatal complications after caesarean section.

The aim of the study was to compare the incidence of postpartum cardiovascular events in parturients with CHD who received generalversus neuraxial anaesthesia. We also investigated the association between anaesthetic technique and neonatal condition after delivery.

\section{Materials and Methods}

Study setting and cohort

This retrospective cohort study included parturients with CHD who gave birth via caesarean section from 1994 to 2019 and whose information was in a database maintained by the Department of Obstetrics and Gynaecology at the National Cerebral and Cardiovascular Centre, in Suita, Osaka, Japan. Parturients with simple congenital arrhythmias or cardiomyopathy were excluded. The institutional ethics committee waived the need for patient consent and approved the study before medical record review began (R19094).

\section{Outcomes}

Cardiovascular events were defined based on the existing literature. ${ }^{14-17}$ The primary outcome was a postpartum cardiovascular event consisting of a composite of all-cause death, new onset or worsening heart failure, pulmonary hypertension, sustained or non-sustained arrhythmia, and thromboembolic complications (myocardial infarction, mechanical valve thrombosis, venous thromboembolism, or cerebrovascular event) that required treatment with intravenous or oral medication or electrical defibrillation. Heart failure that required treatment with only oral diuretics and cardiovascular events that occurred during pregnancy and continued into the postpartum period were not considered primary outcomes.

Secondary outcomes consisted of maternal respiratory events, neonatal all-cause death, neonatal intubation, Apgar score, and umbilical arterial pH. Low Apgar score was defined as Apgar score $<7$ at 1 minute or 5 minutes after delivery. ${ }^{18}$ When a patient was multiple pregnancy, only data on the first newborn was analysed. Composite neonatal outcomes included neonatal intubation and low Apgar score. Primary or secondary outcomes could include events that occurred up to 1 week after caesarean section.

Exposure variables

Anaesthetic technique was analysed as general or neuraxial anaesthesia. We categorized spinal, epidural, or the combination of spinal and epidural anaesthesia as neuraxial anaesthesia and general anaesthesia or the combination of general and epidural anaesthesia as general anaesthesia. Parturients who converted from neuraxial to general anaesthesia were excluded because the aim of the study was to clarify the association between these 2 anaesthetic techniques and outcomes.

Potential confounders

Maternal demographic characteristics included age, multiparity, multiple pregnancy, and body mass index (BMI). BMI was divided into 4 categories based on the existing literature. ${ }^{19}$ Obstetrics characteristics included multiparity, date of caesarean delivery in periods of approximately 5 years to adjust for technical advances in caesarean sections (1994-1999, 2000-2004, 2005-2009, 2010-2014, and 2015-2019), gestational age at delivery categorized into 3 groups based on existing literature: age $<31$ years, age of 32-36 years, and age [?]37 years to adjust for the risk of older age, ${ }^{20}$ type of indication for caesarean section (maternal cardiovascular indication or obstetric indication), ${ }^{21}$ and emergency caesarean delivery status. 
Maternal cardiac demographics included New York Heart Association (NYHA) classification before pregnancy and modified World Health Organization (mWHO) classification for maternal cardiovascular risk. ${ }^{22}$ Two certified experts, an adult cardiologist specializing in caring for obstetric patients and a paediatric cardiologist at our institution, assessed mWHO class for maternal cardiovascular risk, which is the best available assessment model for estimating cardiovascular risk in pregnant women with CHD. ${ }^{23}$

Systemic ventricular ejection fraction $(\mathrm{EF})$ values from the most recent echocardiographic examination before caesarean section were collected. Cardiovascular events during pregnancy included any of the following: heart failure, pulmonary hypertension, sustained or non-sustained arrhythmia, and thromboembolic complications (myocardial infarction, mechanical valve thrombosis, venous thromboembolism, or cerebrovascular event) that required treatment with intravenous or oral medication, electrical defibrillation, or caesarean delivery.

Statistical Analysis

Stata version 15 (College Station, TX, USA) was used for analysis. For baseline patient characteristics, continuous variables are presented as means with standard deviation for normally distributed variables and were compared using the t-test. Non-normally distributed variables are presented as medians (interquartile range) and were compared using the Mann-Whitney U test. Categorical baseline variables were compared using Fisher's exact test.

To account for the heterogeneity of CHD, the association between anaesthetic technique and composite postpartum cardiovascular events was analysed using generalized linear mixed models assuming a binomial distribution (i.e., mixed-effects logistic regression) with a random intercept for each mWHO class for maternal cardiovascular risk. Confounding factors included cardiovascular events during pregnancy, gestational age at delivery, and emergency caesarean section status. The association between anaesthetic technique and composite neonatal outcomes was also analysed using mixed-effects logistic regression with a random intercept for each mWHO classification for maternal cardiovascular risk. Confounding factors included cardiovascular events during pregnancy, gestational age at delivery, birth weight, and emergency caesarean section status. Confounding factors considered to be relevant to primary or secondary outcomes were selected based on existing clinical knowledge. Only parturients with complete information available were included in the analysis.

We also performed sensitivity analyses. First, we added date of caesarean delivery as a confounding factor for both composite maternal and neonatal outcomes. Second, we replaced emergency caesarean status with gestational weeks at delivery or type of indication for caesarean section as potential confounding factors for composite maternal or neonatal outcomes, respectively. Third, we applied inverse probability of treatment weighting (IPTW) to analyse whether anaesthetic technique is associated with composite postpartum cardiovascular events. We used multivariate logistic regression to estimate propensity scores using the following factors: age, BMI, multiparity, multiparous, cardiac events during pregnancy, gestational weeks at delivery, emergency caesarean section status, systemic ventricular EF, date of caesarean delivery, NYHA class before pregnancy and mWHO class for maternal cardiovascular disease. To reduce bias and the amount of missing data, 2 authors abstracted data from electronic medical records from 2008 to 2019 and paper charts from 1994 to 2007.

\section{Results}

During the study period, 263 caesarean sections were performed. General anaesthesia was used in $47(17.9 \%)$ cases and neuraxial anaesthesia was used in $214(81.3 \%)$ cases. Two parturients received both general and spinal anaesthesia. Since the reasons for using both techniques were not clear, these parturients were excluded from the analysis. In the neuraxial anaesthesia group, simple epidural anaesthesia was performed in $24(11 \%)$ cases and spinal anaesthesia with or without epidural anaesthesia in the remaining $190(89 \%)$ cases. The characteristics of the study patients are shown in Table 1. The general anaesthesia group included more parturients with NYHA class III-IV disease, mWHO class IV disease, systemic ventricle $\mathrm{EF}<50 \%$, and emergency caesarean section. In the past, parturients more frequently received general anaesthesia at delivery. More recently, parturients frequently received neuraxial anaesthesia at delivery. At 
delivery, gestational age was lower in the general anaesthesia group than in the neuraxial anaesthesia group. Indications for caesarean section differed significantly between the 2 groups. The amount of missing data was expressed in Table S1.

\section{Postpartum Outcomes}

Postpartum outcomes are shown in Table 2. There were no deaths or respiratory events. Cardiovascular events were more common in the general anaesthesia group $(14.9 \%)$ than in the neuraxial anaesthesia group $(7.9 \%)$, but this difference was not significantly different $(P=0.16)$. Heart failure occurred in parturients with Eisenmenger syndrome ( $\mathrm{n}=1)$, transposition of the great arteries (TGA) repaired with the Jatene procedure $(n=2)$, TGA functionally repaired with the Mustard procedure $(n=1)$, repaired ventricular septal defect (VSD) $(\mathrm{n}=2)$, bicuspid aortic valve (BAV) with aortic regurgitation $(\mathrm{n}=1)$, and unrepaired Epstein anomaly $(\mathrm{n}=1)$. Pulmonary hypertension occurred in parturients with Eisenmenger syndrome $(\mathrm{n}=3)$, repaired atrioventricular septal defect $(\mathrm{n}=1)$, and repaired VSD $(\mathrm{n}=1)$. Arrhythmia occurred in parturients with Fontan circulation $(n=2)$, repaired tetralogy of Fallot $(n=2)$, BAV with aortic dilation $(n=1)$, coarctation of the aorta $(n=1)$, and Kawasaki disease with coronary aneurysm $(n=1)$. Heart failure occurred more frequently in parturients with higher NYHA class or mWHO class. Three parturients in the general anaesthesia group required postpartum transfusion for intraperitoneal bleeding after administration of anticoagulants because of a mechanical valve.

Results from mixed-effects logistic regression models with a random intercept for each mWHO class for maternal cardiovascular risk are shown in Table 3. Compared with neuraxial anaesthesia, general anaesthesia was not significantly associated with composite postpartum cardiovascular events (odds ratio [OR], 1.00; 95\% confidence interval [CI], 0.30-3.29).

\section{Neonatal Outcomes}

Neonatal outcomes are shown in Table S2. Three newborns died of the following causes: intraventricular haemorrhage probably due to maternal anticoagulation with the mechanical valve during pregnancy $(\mathrm{n}=1)$, after surgery to correct neonatal CHD $(n=1)$; immediately after birth due to severe neonatal CHD $(n=1)$. The general anaesthesia group had a significantly higher percentage of neonatal intubations $(53.2 \%$ vs. $7.0 \%$, $P<0.001)$, Apgar scores $<7$ at 1 or 5 minutes $(57.4 \%$ vs. $2.8 \%, P<0.001)$ and composite neonatal outcome $(63.4 \%$ vs. $8.9 \%, \mathrm{P}<0.001)$. Mixed-effects logistic regression models with a random intercept for each mWHO class for maternal cardiovascular risk revealed that general anaesthesia is significantly associated with a higher rate of composite neonatal outcomes (OR, 13.3; 95\% CI, 5.52-32.0) (Table 4).

Sensitivity analysis

In the sensitivity analysis with date of delivery as a confounding factor, maternal outcomes remained not significantly associated with general anaesthesia (OR, 0.76; 95\% CI, 0.19-3.00). Results were also unchanged in the sensitivity analysis where emergency caesarean section status was changed to gestational weeks at delivery as a potential confounding factor in the model (OR, 0.51; 95\% CI, 0.24-1.11). Regarding neonatal outcomes, in the sensitivity analysis with date of delivery as a confounding factor, composite neonatal outcomes remained associated with general anaesthesia (OR, 8.84; 95\% CI, 3.54-22.1). Results were also unchanged in the sensitivity analysis where emergency caesarean section status was changed to type of indication for caesarean section as a potential confounding factor in the model (OR, 12.8; 95\% CI, 5.4930.0). In the propensity score analysis using IPTW, composite postpartum cardiovascular events remained not significantly associated with general anaesthesia (OR, 0.51; 95\% CI, 0.12-2.20) and composite neonatal outcomes remained significantly associated with general anaesthesia (OR, 4.56; 95\% CI, 1.48-14.0). The c-index for the logistic regression model that was used to calculate propensity scores was 0.90 .

\section{Discussion}

This study compared postpartum cardiovascular events and neonatal outcomes in parturients with CHD by anaesthetic technique (generalversus neuraxial anaesthesia). We showed that general anaesthesia is not 
significantly associated with composite postpartum cardiovascular events but is significantly associated with a higher rate of composite neonatal outcomes based on mixed-effects logistic regression.

Our main results are not consistent with a prior systematic review of case reports that suggest higher maternal mortality with general anaesthesia and improved outcomes with neuraxial anaesthesia, but this systematic review could not exclude selection bias due to sicker parturients receiving general anesthesia. ${ }^{13}$ The mixed-effects logistic regression models which adjusted for major confounders make the present study more reliable. During the 25 years of this study, the overall safety of general anaesthesia for caesarean section has improved dramatically in parturients without CHD, as outlined in a recent review. ${ }^{24}$ In addition, gestational age at delivery is a known confounder due to the larger impact of pregnancy on hemodynamic during the second or third trimester than during the first trimester. ${ }^{3}$ Therefore, we conducted sensitivity analyses that included date of caesarean delivery or gestational age at delivery as confounders and propensity score analysis using the IPTW approach. Results of the sensitivity analyses showed the robustness of the primary results.

Regarding neonatal outcomes, the OR for the association between general anaesthesia and adverse composite neonatal outcomes was high. Sensitivity analyses that considered date of caesarean delivery and other possible confounders resulted in higher ORs, thus supporting the primary results. These results were not consistent with a previous systematic review that showed no significant difference between 2 anaesthetic techniques in terms of neonatal outcomes. ${ }^{25}$ The differences may be attributed to the manner of general anaesthesia induction in parturients with CHD. Induction of general anaesthesia in parturients with high cardiac risk took longer than in the general population. Blunting the hemodynamic response at intubation and during surgery is crucial for maintaining hemodynamic stability in parturients with cardiac disease. Especially during induction, titration of anaesthetics and analgesics can achieve hemodynamic stability more easily than rapid sequence induction. Adequate doses of anaesthetics and analgesics to prevent noxious responses to intubation in parturients often cause neonatal depression. Therefore, neonatal outcomes were worse in the general anaesthesia group. However, an adequate dose for a parturient might outweigh the risk of neonatal depression, because fetal well-being depends on the maintenance of maternal hemodynamic stability and the effects of anaesthetics and opioid analgesics on the fetus could be reversible. ${ }^{26}$

The choice of neuraxial or general anaesthesia should be tailored for each individual parturient in order to maintain hemodynamic stability after considering the risks and benefits of both anaesthetic techniques. ${ }^{27}$ Since we did not find significant associations between increased maternal risk and anaesthetic technique, both anaesthetic techniques may be acceptable, especially general anaesthesia when there are contraindications to neuraxial anaesthesia, emergency surgery, or need for invasive monitoring. Moreover, the choice of anaesthetic technique should be made with consideration that general anaesthesia is associated with a higher risk of neonatal intubation or lower Apgar scores based on our results.

This study had the following limitations. First, because this study was retrospective in design, there might be residual confounding. However, most previous studies investigating the association between anaesthetic technique and postpartum and neonatal outcomes were case series. Due to the limited population of pregnant women with CHD, it is not feasible to perform randomized controlled trials to evaluate the superiority of the anaesthetic techniques. Therefore, we performed a retrospective chart review. Second, information about dose and type of drugs used, hemodynamic data, and type of invasive monitoring used during the perioperative period were not included as potential confounders due to lack of data. Third, due to the low rate of maternal and neonatal events, we could not avoid evaluating composite outcomes, which might make the results difficult to interpret.

\section{Conclusion}

We showed that anaesthetic technique is not significantly associated with postpartum composite cardiovascular events and that general anaesthesia is significantly associated with increased need for intubation and lower Apgar scores in neonates.

\section{Disclosure of interest}


The authors declare that they have no conflicts of interest. Completed disclosure of interest forms are available to view online as supporting information.

\section{Contribution to authorship}

AT, KY, YK and EK conceived and designed this study. AT and YK collected the data. KN, MN and SO performed the statistical analyses for this study. AT and KY wrote the manuscript. RT, CK, JY and YO reviewed the manuscript. All authors read and approved the final manuscript.

\section{Details of ethics approval}

Ethical approval was granted by the National Cerebral and Cardiovascular Centre Research and Ethics Committee (Study No. R19094) in January 2020.

\section{Funding}

No external funding was used in the conduct of this study.

\section{Acknowledgement}

We thank ZENIS (https://www.zenis.co.jp/) for editing the draft version of this manuscript.

\section{References}

1. Whittemore R, Hobbins JC, Engle MA. Pregnancy and its outcome in women with and without surgical treatment of congenital heart disease. Am J Cardiol. 1982 Sep;50(3):641-51.

2. van der Linde D, Konings EE, Slager MA, Witsenburg M, Helbing WA, Takkenberg JJ, et al. Birth prevalence of congenital heart disease worldwide: a systematic review and meta-analysis. Journal of the American College of Cardiology. 2011 Nov 15;58(21):2241-7.

3. Sanghavi M, Rutherford JD. Cardiovascular physiology of pregnancy. Circulation. 2014 Sep $16 ; 130(12): 1003-8$.

4. Hrycyk J, Kaemmerer H, Nagdyman N, Hamann M, Schneider K, Kuschel B. Mode of Delivery and Pregnancy Outcome in Women with Congenital Heart Disease. PLoS One. 2016;11(12):e0167820.

5. Hayward RM, Foster E, Tseng ZH. Maternal and Fetal Outcomes of Admission for Delivery in Women With Congenital Heart Disease. JAMA Cardiol. 2017 Jun 1;2(6):664-71.

6. Bucklin BA, Hawkins JL, Anderson JR, Ullrich FA. Obstetric anesthesia workforce survey: twenty-year update. Anesthesiology. 2005 Sep;103(3):645-53.

7. Djabatey EA, Barclay PM. Difficult and failed intubation in 3430 obstetric general anaesthetics. Anaesthesia. 2009 Nov;64(11):1168-71.

8. Kodali BS, Chandrasekhar S, Bulich LN, Topulos GP, Datta S. Airway changes during labor and delivery. Anesthesiology. 2008 Mar;108(3):357-62.

9. Maxwell BG, El-Sayed YY, Riley ET, Carvalho B. Peripartum outcomes and anaesthetic management of parturients with moderate to complex congenital heart disease or pulmonary hypertension*. Anaesthesia. 2013 Jan;68(1):52-9.

10. Goldszmidt E, Macarthur A, Silversides C, Colman J, Sermer M, Siu S. Anesthetic management of a consecutive cohort of women with heart disease for labor and delivery. Int J Obstet Anesth. 2010 Jul;19(3):266-72.

11. Hidano G, Uezono S, Terui K. A retrospective survey of adverse maternal and neonatal outcomes for parturients with congenital heart disease. Int J Obstet Anesth. 2011 Jul;20(3):229-35. 
12. Warrick CM, Hart JE, Lynch AM, Hawkins JA, Bucklin BA. Prevalence and descriptive analysis of congenital heart disease in parturients: obstetric, neonatal, and anesthetic outcomes. Journal of clinical anesthesia. 2015 Sep;27(6):492-8.

13. Bédard E, Dimopoulos K, Gatzoulis MA. Has there been any progress made on pregnancy outcomes among women with pulmonary arterial hypertension? Eur Heart J. 2009 Feb;30(3):256-65.

14. Khairy P, Ouyang DW, Fernandes SM, Lee-Parritz A, Economy KE, Landzberg MJ. Pregnancy outcomes in women with congenital heart disease. Circulation. 2006 Jan 31;113(4):517-24.

15. Drenthen W, Boersma E, Balci A, Moons P, Roos-Hesselink JW, Mulder BJ, et al. Predictors of pregnancy complications in women with congenital heart disease. Eur Heart J. 2010 Sep;31(17):2124-32.

16. Siu SC, Sermer M, Colman JM, Alvarez AN, Mercier LA, Morton BC, et al. Prospective multicenter study of pregnancy outcomes in women with heart disease. Circulation. 2001 Jul 31;104(5):515-21.

17. Ruys TP, Roos-Hesselink JW, Hall R, Subirana-Domènech MT, Grando-Ting J, Estensen M, et al. Heart failure in pregnant women with cardiac disease: data from the ROPAC. Heart. 2014 Feb;100(3):231-8.

18. Razaz N, Cnattingius S, Joseph KS. Association between Apgar scores of 7 to 9 and neonatal mortality and morbidity: population based cohort study of term infants in Sweden. BMJ. 2019 May 7;365:11656.

19. Cnattingius S, Bergstrom R, Lipworth L, Kramer MS. Prepregnancy weight and the risk of adverse pregnancy outcomes. The New England journal of medicine. 1998 Jan 15;338(3):147-52.

20. Cnattingius S, Norman M, Granath F, Petersson G, Stephansson O, Frisell T. Apgar Score Components at 5 Minutes: Risks and Prediction of Neonatal Mortality. Paediatr Perinat Epidemiol. 2017 Jul;31(4):328-37.

21. Warnes CA. Pregnancy and Delivery in Women With Congenital Heart Disease. Circ J. 2015;79(7):141621.

22. Regitz-Zagrosek V, Roos-Hesselink JW, Bauersachs J, Blomstrom-Lundqvist C, Cifkova R, De Bonis M, et al. 2018 ESC Guidelines for the management of cardiovascular diseases during pregnancy. Eur Heart J. 2018 Sep 7;39(34):3165-241.

23. Balci A, Sollie-Szarynska KM, van der Bijl AG, Ruys TP, Mulder BJ, Roos-Hesselink JW, et al. Prospective validation and assessment of cardiovascular and offspring risk models for pregnant women with congenital heart disease. Heart. 2014 Sep;100(17):1373-81.

24. Sumikura H, Niwa H, Sato M, Nakamoto T, Asai T, Hagihira S. Rethinking general anesthesia for cesarean section. Journal of anesthesia. 2016 Apr;30(2):268-73.

25. Afolabi BB, Lesi FE. Regional versus general anaesthesia for caesarean section. Cochrane Database Syst Rev. 2012 Oct 17;10:CD004350.

26. Moir DD. Anaesthesia for Caesarean section. An evaluation of a method using low concentrations of halothane and 50 per cent of oxygen. British journal of anaesthesia. 1970 Feb;42(2):136-42.

27. Bishop L LA, English K. Adult congenital heart disease and pregnancy. British Jornal of Anaesthesia Education. 2018;18(1):23-9.

Table 1. Patient characteristics

\begin{tabular}{llll}
\hline & Neuraxial anesthesia $(\mathrm{n}=214)$ & General anesthesia $(\mathrm{n}=47)$ & $P$ value \\
\hline Age (years) & $31.6 \pm 5.1$ & $31.0 \pm 4.9$ & 0.42 \\
Multiparity & $86(40.2 \%)$ & $20(42.6 \%)$ & 0.87 \\
Multiple pregnancy & $10(4.7 \%)$ & $1(2.1 \%)$ & 0.70 \\
Body mass index $\left(\mathrm{kg} \mathrm{m}^{-2}\right)<20$ & $74(36.3 \%)$ & $20(50.0 \%)$ & 0.18 \\
$20-24.9$ & $111(54.4 \%)$ & $20(50.0 \%)$ &
\end{tabular}




\begin{tabular}{|c|c|c|c|}
\hline & Neuraxial anesthesia $(\mathrm{n}=214)$ & General anesthesia $(n=47)$ & $P$ value \\
\hline $25.0-29.9$ & $9(4.4 \%)$ & 0 & $? \gtreqless ?$ \\
\hline 30 & $10(4.9 \%)$ & 0 & \\
\hline Date (year of caesarean section) 1994-1999 & $11(5.1 \%)$ & $11(23.4 \%)$ & $<0.001$ \\
\hline $2000-2004$ & $18(8.4 \%)$ & $11(23.4 \%)$ & \\
\hline $2005-2009$ & $46(21.5 \%)$ & $10(21.3 \%)$ & \\
\hline $2010-2014$ & $59(27.6 \%)$ & $11(23.4 \%)$ & \\
\hline 2015-2019 & $80(37.4 \%)$ & $4(8.5 \%)$ & \\
\hline Pre-pregnancy NYHA class I & $188(87.9 \%)$ & $30(63.8 \%)$ & $<0.001$ \\
\hline II & $24(11.2 \%)$ & $14(29.8 \%)$ & \\
\hline III & $2(0.9 \%)$ & $3(6.4 \%)$ & \\
\hline IV & 0 & 0 & \\
\hline mWHO class I & $59(27.6 \%)$ & $8(17.0 \%)$ & $<0.001$ \\
\hline II & $69(32.2 \%)$ & $12(25.5 \%)$ & \\
\hline II-III & $57(26.6 \%)$ & $12(25.5 \%)$ & \\
\hline III & $28(13.1 \%)$ & $7(14.9 \%)$ & \\
\hline IV & $1(0.5 \%)$ & $8(17.0 \%)$ & \\
\hline Systemic ventricular $\mathrm{EF}<50$ & $9(4.3 \%)$ & $6(14.3 \%)$ & 0.029 \\
\hline Gestational age at delivery (weeks) [?]31 & $5(2.3 \%)$ & $7(14.9 \%)$ & $<0.001$ \\
\hline $32-36$ & $33(15.4 \%)$ & $18(38.3 \%)$ & $? i ?$ \\
\hline 37 & $176(82.2 \%)$ & $22(46.8 \%)$ & \\
\hline Type of indication for caesarean section & & & 0.001 \\
\hline Maternal cardiovascular indication & $19(8.9 \%)$ & $13(27.7 \%)$ & \\
\hline Obstetric indication & $195(91.1 \%)$ & $34(72.3 \%)$ & \\
\hline Emergency caesarean section & $74(34.6 \%)$ & $30(63.8 \%)$ & $<0.001$ \\
\hline
\end{tabular}

EF, ejection fraction; mWHO class, modified World Health Organization class of maternal cardiovascular risk; NYHA, New York Heart Association.

Data are expressed as n (\%) for categorical variables or means \pm standard deviation for continuous variables. Categorical variables were compared using Fisher's exact test and continuous variables were compared using the t-test.

Table 2. Postpartum outcomes

\begin{tabular}{llll}
\hline & $\begin{array}{l}\text { Neuraxial anesthesia } \\
(\mathrm{n}=214)\end{array}$ & $\begin{array}{l}\text { General anesthesia } \\
(\mathrm{n}=47)\end{array}$ & $P$ value \\
\hline All-cause death & 0 & 0 & \\
Cardiovascular event & $17(7.9 \%)$ & $7(14.9 \%)$ & 0.16 \\
Heart failure & 7 & 5 & \\
Pulmonary hypertension & 3 & 2 & \\
Arrhythmia & 7 & 0 & \\
Thromboembolic & 0 & 0 & \\
complication & & 0 & \\
Respiratory event & 0 & $4(8.5 \%)$ & \\
Other & $3(1.4 \%)$ &
\end{tabular}

Data are expressed as n (\%) and were compared using Fisher's exact test.

* Other included transfusion $(n=4)$, depression $(n=1)$, and pregnancy-induced hypertension $(n=2)$. 
Table 3. Association between cardiovascular events after caesarean section with anesthetic technique based on mixed-effects logistic regression models with random intercepts for each mWHO class for maternal cardiovascular risk

\begin{tabular}{lllll}
\hline & Unadjusted odds ratio (95\% CI) & $P$ value & Adjusted odds ratio (95\% CI) & $P$ value \\
\hline General anesthesia & $1.31(0.43-4.04)$ & 0.64 & $1.00(0.30-3.29)$ & 0.99 \\
Cardiac events during pregnancy & $5.47(2.11-14.12)$ & $<0.001$ & $5.34(2.05-13.9)$ & 0.001 \\
Emergency caesarean section & $1.41(0.58-3.44)$ & 0.45 & $1.23(0.48-3.14)$ & 0.66 \\
\hline
\end{tabular}

mWHO, modified World Health Organization; CI, confidence interval.

${ }^{*}$ Relative to neuraxial anesthesia

Table 4. Association between composite neonatal outcomes ${ }^{*}$ after caesarean section and anesthetic technique based on mixed-effects logistic regression models with random intercepts for each mWHO class for maternal cardiovascular risk

\begin{tabular}{lllll}
\hline & Unadjusted odds ratio $(95 \% \mathrm{CI})$ & $P$ value & Adjusted odds ratio $(95 \% \mathrm{CI})$ & $P$ valu \\
\hline General anesthesia $^{* *}$ & $18.1(8.41-38.9)$ & $<0.001$ & $13.3(5.52-32.0)$ & $<0.001$ \\
Cardiac event during pregnancy & $312(1.44-6.76)$ & 0.004 & $1.29(0.47-3.50)$ & 0.62 \\
Gestational age at delivery category & $0.16(0.09-0.28)$ & $<0.001$ & $0.28(0.11-0.69)$ & 0.006 \\
Birth weight (per 100 g) & $0.83(0.77-0.89)$ & $<0.001$ & $0.96(0.86-1.06)$ & 0.40 \\
Emergency caesarean section & $0.66(0.52-0.85)$ & 0.001 & $0.90(0.38-2.10)$ & 0.80 \\
\hline
\end{tabular}

mWHO, modified World Health Organization; CI, confidence interval.

${ }^{*}$ Composite neonatal outcome includes need for intubation and Apgar score $<7$ at 1 minute or 5 minutes.

${ }^{* *}$ Relative to neuraxial anesthesia 